\title{
BMJ Open National cross-sectional survey of 1.14 million NHS staff SARS-CoV-2 serology tests: a comparison of NHS staff with regional community seroconversion rates
}

Cordelia E M Coltart (D) ,1,2 David Wells, ${ }^{3}$ Esther Sutherland, ${ }^{4}$ Aidan Fowler ${ }^{2,3}$

To cite: Coltart CEM, Wells D, Sutherland E, et al. National cross-sectional survey of 1.14 million NHS staff SARS-CoV-2 serology tests: a comparison of NHS staff with regional community seroconversion rates. BMJ Open 2021;0:e049703. doi:10.1136/ bmjopen-2021-049703

- Prepublication history for this paper is available online. To view these files, please visit the journal online (http://dx.doi. org/10.1136/bmjopen-2021049703).

Received 17 February 2021 Accepted 29 April 2021

Check for updates

(c) Author(s) (or their employer(s)) 2021. Re-use permitted under CC BY-NC. No commercial re-use. See rights and permissions. Published by BMJ.

${ }^{1}$ Institute for Global Health, University College London, London, UK

${ }^{2}$ Chief Medical Officer's Office, Department of Health and Social Care, London, UK

${ }^{3}$ COVID-19 Testing cell, NHS England and NHS Improvement, London, UK

${ }^{4}$ COVID-19 Infection Survey, Office for National Statistics, Newport, UK

Correspondence to Dr Aidan Fowler; aidan.fowler@dhsc.gov.uk and Dr Cordelia E M Coltart; cordelia.coltar@@ucl.ac.uk

\section{ABSTRACT}

Objectives An initial report of findings from 1.14 million SARS CoV-2 serology tests in National Health Service (NHS) staff to compare NHS staff seroconversion with community seroconversion rates at a regional level. Design A national cross-sectional survey.

Setting A SARS-CoV-2 antibody testing programme offered across all NHS Trusts.

Participants 1.14 million NHS staff.

Intervention SARS-CoV-2 antibody testing.

Primary and secondary outcome measures SARS-CoV-2 antibody testing was used to estimate the seroprevalence of SARS-CoV-2 in NHS staff by region, compared with community seroprevalence as determined by the COVID-19 Infection Survey (Office for National Statistics). We also explored seroprevalence trends by regional COVID-19 activity, using regional death rates as a proxy for COVID-19 'activity'.

Results 1146310 tests were undertaken on NHS staff between 26 May and 31 August 2020.186 897 NHS tests were positive giving a seroconversion rate of $16.3 \%$ (95\% $\mathrm{Cl} 16.2 \%$ to $16.4 \%$ ), in contrast to the national community seroconversion rate of $5.9 \%$ (95\% $\mathrm{Cl} 5.3 \%$ to $6.6 \%)$. There was significant geographical regional variation, which mirrored the trends seen in community prevalence rates. NHS staff were infected at a higher rate than the general population (OR 3.1, $95 \% \mathrm{Cl} 2.8$ to 3.5). NHS seroconversion by regional death rate suggested a trend towards higher seroconversion rates in the areas with higher COVID-19 'activity'.

Conclusions This is the first cross-sectional survey assessing the risk of COVID-19 disease in healthcare workers at a national level. It is the largest study of its kind. It suggests that NHS staff have a significantly higher rate of COVID-19 seroconversion compared with the general population in England, with regional variation across the country which matches the background population prevalence trends. There was also a trend towards higher seroconversion rates in areas which had experienced high COVID-19 clinical activity. This work has global significance in terms of the value of such a testing programme and contributing to the understanding of healthcare worker seroconversion at a national level.

\section{INTRODUCTION}

In December 2019, the first cases of an unknown disease were reported in Wuhan,
Strengths and limitations of this study

- The key strength of this report is the large sample size ( $n>1.14$ million) which is substantially larger than similar reports and the national coverage.

- This is a technical report and not a research project which results in many limitations (outlined in the Discussion section).

- This report is restricted to data from a period during the first wave of the pandemic (May to August 2020)

- Due to the limitations discussed, this report is not able to answer crucial questions on transmission dynamics of hospital-associated infections, including drivers of infection, direction of transmission, risk factors for infection and at-risk groups.

China. The causative organism was subsequently identified to be a novel coronavirus, SARS-CoV-2, which results in a clinical disease called COVID-19. COVID-19 causes a wide spectrum of presentations in humans, varying from asymptomatic infection to a mild/non-specific predominantly respiratory infection, to severe disease with respiratory failure, multiorgan failure and death. Cases quickly spread worldwide and COVID-19 was classified as a global pandemic on 11 March 2020. As of 9 November 2020, there had been approximately 50 million reported cases and 1.25 million deaths as a result of COVID-19 in 214 countries. ${ }^{1}$ In England, there had been 1.02 million confirmed cases and 43191 deaths (49044 deaths in the UK). ${ }^{2}$ The impact on healthcare and society has been profound, both in England and globally. Mitigating future waves and epidemics is a public health priority globally.

In many epidemics, healthcare workers (HCWs) have been reported to be at increased risk of occupational infection and have been suggested to be a source of onward 
transmission to other HCWs, patients, and within their community. This is true in both respiratory and nonrespiratory infectious outbreaks. For example, $21 \%$ of the 2003 SARS epidemic cases globally were thought to involve $\mathrm{HCWs}^{3}$ with a higher proportion (between $37 \%$ and $63 \%$ of suspected cases) reported in highly affected countries ${ }^{4}$ and much of the disease worldwide associated with hospital-based outbreaks. ${ }^{5}{ }^{6} \mathrm{~A}$ meta-analysis of the occupational risk of influenza A (H1N1) infection among HCWs during the 2009 influenza pandemic showed they were at increased risk of infection (OR 2.08 (95\% CI 1.73 to 2.51$))^{7}$; a wider systematic review of HCWs' risk of influenza compared with other healthy adults in non-healthcare settings (across 60 years and 97 influenza seasons, $\mathrm{n}=58245$ ) showed a significantly higher risk of infection in HCWs (incidence rate ratio of 3.4 (95\% CI 1.2 to 5.7 ) in unvaccinated HCWs, and 5.4 (95\% CI 2.8 to 8.0 ) in vaccinated $\mathrm{HCWs})^{8}$; and HCWs were reported to have a relative risk of acquiring Ebola Virus Disease (during the 2013-2016 epidemic) of more than 100 times compared to the general population. ${ }^{9}{ }^{10}$ This trend is also seen in early reports of COVID-19. In Wuhan, China, where the incidence of infection was higher in HCWs than the general public. ${ }^{11}$ Self-reported data on the COVID-19 Symptom Study app in the UK and USA suggested that frontline HCWs had at least a threefold increased risk of infection compared with non-HCWs ${ }^{12}$; and HCW SARS-CoV-2 seroprevalence in a large acute care hospital in Sweden showed an increased occupation health risk with higher seroprevalence $(19.1 \%)$ in HCWs than the regional rate. ${ }^{13}$

Despite the clear risk to HCWs and the risk they pose to patients, to one another and to those with whom they have contact in the community, there remains a lack of comprehensive data on HCW infection, exposure and seroconversion. ${ }^{12}$ Nosocomial transmission (transmission of infection related to a healthcare setting) has been postulated as playing a key role contributing to the current pandemic both internationally and in England, although the exact contribution remains unknown. ${ }^{14-17}$ Although the importance of this transmission pathway remains unclear, modelling has suggested that at least $10 \%(95 \%$ CI $4 \%$ to $15 \%)$ of all COVID-19 infections in England between 26 April and 7 June 2020 were in HCWs. ${ }^{14}$

Serology (or antibody) tests can be used to determine an antibody response in the blood of those who have previously been infected with SARS-CoV-2, including those with asymptomatic and mild infection. The serology test should be taken at least 2-3 weeks after infection to allow enough time for an immune response to develop and be reliably detected. However, it remains positive for at least several months, although the exact window is unclear and is an active area of current research. ${ }^{18}$

This is an initial report of findings from 1.14 million serology tests in National Health Service (NHS) staff and is the largest study of its kind. This work will inform NHS planning for subsequent waves of the COVID-19 pandemic in England, and it has global significance in terms of the value of such a testing programme and the conclusions that can be drawn from this study.

\section{METHODS}

In May 2020, the Secretary of State for Health and Social Care made a commitment to offer a SARS-CoV-2 antibody test to all NHS staff who wanted it as part of the COVID-19 response. Thus, a rapid testing programme was established. This report uses data from NHS England and Improvement (NHS E\&I), obtained routinely as part of this testing and surveillance programme.

The antibody testing programme began data collection on 26 May 2020 and the data set used in this report includes tests taken from that date up to, and including, 31 August 2020. Each employing trust was tasked to ensure every employee was offered a test and the uptake of these tests was reported on a daily basis to NHS England and the Department of Health and Social Care. A total of 1146310 tests were undertaken on NHS Trust staff. This represents an approximate coverage of $89 \%$ of the total 1.29 million reported NHS Trust staff. ${ }^{19}$ The tests were provided on an appointment basis and data were recorded against human resources databases. No repeat testing was agreed. Therefore, it was anticipated that duplication of testing would be minimal. This study includes any sample reported as being sent from an NHS Trust or Foundation Trust, including samples obtained from acute, community and mental health trusts. Samples were submitted from 195 out of 218 NHS Trusts. Of note, primary care samples were excluded.

All data were anonymised and as this is a technical report based on the data available, not a research project, individual consent was not obtained. The only metadata included in this report is the region where the sample originated. For the purpose of analysis, the referral source of samples was assumed to be the employing organisation and used by NHS E\&I to determine the region of origin.

\section{Serology testing}

Blood samples were drawn using standard venepuncture techniques. Prepared serum samples were then analysed for SARS-CoV-2 antibodies using a range of commercially available immunoassays, depending on the laboratory where the sample was processed. The assays included those targeted at antibiodies directed against the spike protein (Diasorin and Ortho Clinical diagnostics), the nucleocapsid protein (Abbott and Roche), and a range of immunoglobulin proteins (IgG-Abbott and Diasorin), or total immunoglobulins (IgM and IgG-Ortho Clinical diagnostics and Roche). Results were reported as positive, negative or equivocal at cut-offs defined by the manufacturer. Results were reported into the local laboratory information system. Laboratories submit aggregated antibody test data on a daily basis (midnight to midnight) to NHS E\&I, where it was logged via the Pathology Laboratory 
Activity and Capacity Electronic Reporting Systems, an online portal for collection of COVID-19 test data.

\section{Comparator community data}

To contextualise the findings in NHS staff, data from the COVID-19 Infection Survey (CIS) run by the Office for National Statistics (ONS) were used. This study aims to determine COVID-19 infection in the general population and produces (among other parameters) estimates of the number and percentage of people testing positive for antibodies in the community population in England (ie, background seroprevalence). It includes a survey as well as reverse transcription (RT)-PCR viral testing and serology testing in a subset of the cohort. ${ }^{20}$ This study uses summary data provided by ONS regarding the total number of serology tests undertaken and the number which were positive, by geographical region in England. It is limited to the working age population only (ie, those aged 18-65years old). These data were collected between 26 April and 8 September 2020 to provide a comparable time frame.

\section{Statistical analyses}

All statistical analyses were undertaken using Stata V.14 (Stata Corp, College Station, Texas, USA). Odds Ratios (OR) were calculated to determine the likelihood of infection in NHS staff compared with background community population seroprevalence rates. All analyses are unweighted. Furthermore, we examined whether there were any trends in seroprevalence rates by regional COVID-19 activity, using regional COVID-19 death rates as a proxy for COVID-19 activity. ${ }^{2}$

\section{Patient and public involvement}

No patients were involved, this is a working report and not a formal research study (please see the Discussion section).

\section{RESULTS}

The NHS staff serology testing programme undertook 1146310 tests on NHS staff from 195 NHS Trusts between 26 May and 31 August 2020. A total of 186897 tests were positive. The overall proportion of positive COVID-19 serology tests in NHS staff is $16.3 \%$ (95\% CI $16.2 \%$ to $16.4 \%$ ). However, there is significant geographical regional variation, with the highest rates in London $(23.9 \%)$ and lowest in the South West $(8.9 \%)$ (table 1). The regional variation is statistically significant across all regions $\left(\mathrm{p}<0.001, \mathrm{X}^{2}\right.$ test $)$.

Comparing NHS staff data with data from the community population (CIS, ONS), the NHS regional trends mirror the regional variation in community prevalence rates reported (ie, the rankings for seroprevalence match, with the minor exception of the East of England and North West alternating between second and third highest when comparing the NHS with ONS data). However, across all regions, the NHS seroprevalence rates show

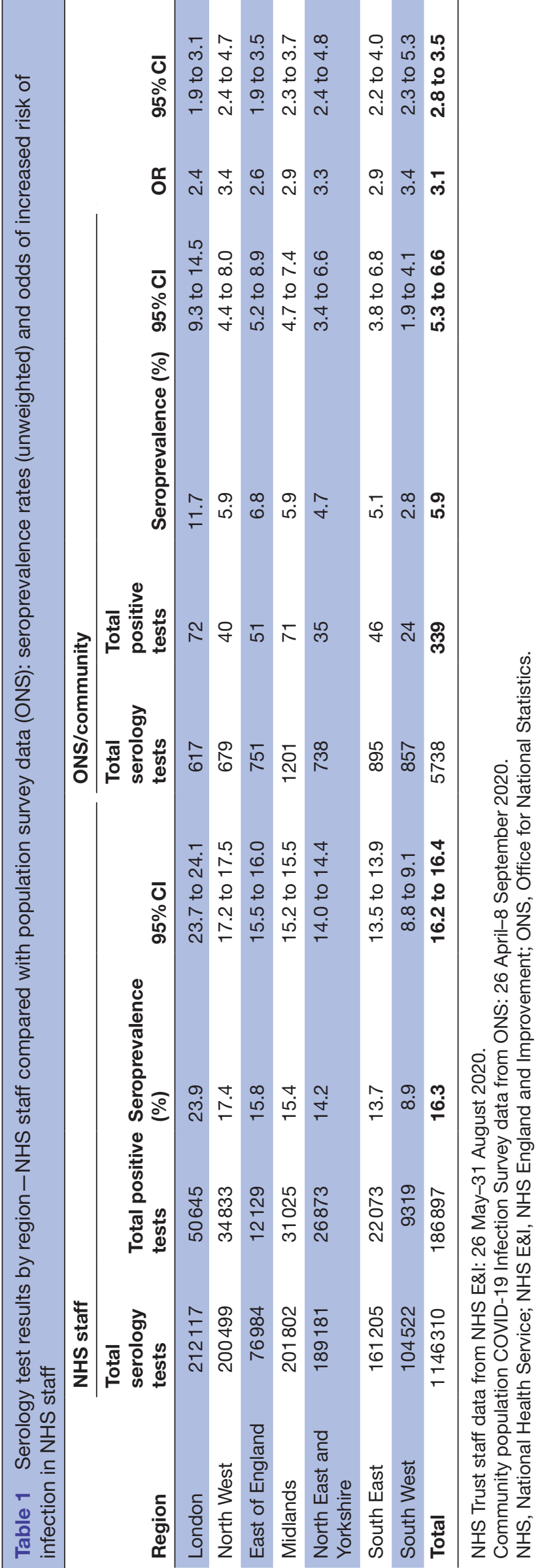




\section{Regional NHS seroprevalence by Death rate (per 100,000 population)}

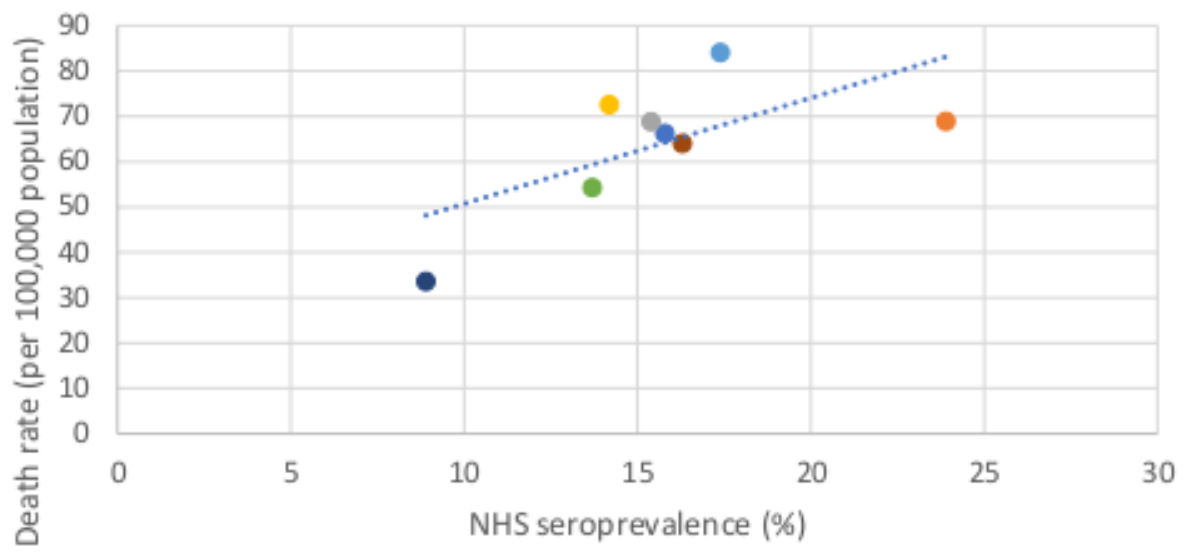

\section{Key: \\ North West \\ London \\ National average \\ East of England \\ MIdlands \\ Nouth East \\ South West}

Figure 1 Scatter plot of NHS staff seroprevalence by 'COVID-19 activity', as determined by regional death rate (per 100000 population). NHS, National Health Service.

a highly significant difference when compared with the community population level seroprevalence rates (with no overlapping CIs between the two groups).

ORs were calculated to determine the likelihood of infection in NHS staff compared with background community population seroprevalence rates. NHS staff were infected at a higher rate than the general population (OR 3.1, 95\% CI 2.8 to 3.5) (table 1). Of note, the community data are based on a relatively small sample with wide CIs.

The trend between NHS seroconversion and regional death rate (per 100000 population) as a proxy for regional COVID-19 activity was analysed. This suggested a possible trend towards higher seroconversion rates in the areas with high death rates (figure 1). Given this analysis is limited to 8 data points, the statistical significance of this trend has not been calculated.

\section{DISCUSSION}

To our knowledge, this report is the first cross-sectional survey assessing the risk of COVID-19 infection in HCWs at a national level. The report suggests that NHS staff are at higher risk of COVID-19 compared with the general population in England: the overall proportion of positive SARS-CoV-2 serology tests in NHS staff is $16.3 \%$, while for the general population in England the equivalent seroprevalence is $5.9 \%$. There was regional variation across the country and areas which experienced a high prevalence of COVID-19 in the general population, for example London, also showed an increased prevalence in NHS staff (11.7\% vs $23.9 \%$ respectively). Throughout the country, NHS staff showed a significantly higher rate of infection than the community as a whole, with an Odds Ratio of having a positive test of 3.1 (95\% CI 2.8 to 3.5). There was also a trend towards higher seroconversion rates in areas which had experienced high COVID-19 clinical activity (defined by regional COVID-19 death rate as a proxy marker).

\section{HCW infection}

Comprehensive comparator data on HCW infections are limited. The estimates of risk of infection for HCWs are variable and the findings conflicting: most reports suggest a higher level of infection in HCWs compared with the general population (both by RT-PCR and antibody testing), ${ }^{13} 21$ although other studies have suggested that infection rates in HCWs reflect the community incidence rate. ${ }^{22}$ Of note, this report suggests that the rates of infection in NHS staff are not as high as some previous estimates reported from England, notably social medial reports of seroprevalence up to $60 \%{ }^{23}$ Studies have estimated seroprevalence to be between $15 \%$ and $45 \%$ in HCWs in patient-facing roles and $3 \%-25 \%$ overall. ${ }^{21}{ }^{24-26}$ These rates are thought to vary by specialty, ${ }^{27}$ but the infection rates between different subgroups vary by report. Contrary to expectations, one UK study suggests that intensive care clinicians had the lowest infection rate, while cleaners had the highest rate, along with acute and general medicine clinicians and those of black, Asian, and minority ethnic background. ${ }^{26}$ Overall, the highest reported seroprevalence was from a central London hospital during a 1-month period at the peak of the epidemic (March-April 2020) where 45\% of clinical staff had positive antibody tests and $21 \%$ tested RT-PCR positive for current infection. ${ }^{21}$ In comparison, European studies show an HCW seroprevalence rate of $19.1 \%$ in Sweden, ${ }^{13} 4 \%$ in Denmark ${ }^{28}$ and $13.9 \%$ in Italy. ${ }^{29}$ Several studies noted that a high proportion of HCWs had asymptomatic or mildly symptomatic infections, including English data suggesting that 57\% and $81 \%$ of PCR-positive HCWs had few or no symptoms. ${ }^{21} 30$ 
This is concerning in terms of unrecognised transmission risk. Furthermore, unpublished preliminary modelling data from Public Health England estimated that 20\% of inpatient infections, and up to $73 \%$ of HCW infections, were hospital acquired. ${ }^{31}$ Unpublished genomic data suggest that staff-to-staff transmission is likely to play a more significant role than patient-to-staff or vice versa in the hospital setting. ${ }^{32}$

There are numerous reasons why HCWs are at high risk of infection including a higher contact rate with infected individuals, higher viral exposure to SARS-CoV-2 while caring for severely ill infectious patients, contact with vulnerable populations (with comorbidities and risk factors for COVID-19 infection), difficulty in distancing while providing clinical care to patients and within social spaces in hospitals, personal protective equipment (PPE) shortages, variable use of PPE particularly in the early stages of the pandemic, working in indoor settings, and the inability to stay at home during lockdown due to work commitments, which is associated with an increased number of contacts and risks. Indeed, as outlined above, the role of staff-to-staff transmission appears to be particularly important in transmission chains (from both modelling and genomic data).${ }^{15-17} 3132$ Furthermore, the cumulative repeat exposure of HCWs over time is likely to be high, which may lead to some immunity generation and thus, potentially render them at higher risk of asymptomatic reinfection. This is particularly important as we endure further waves, due to the increased risk of onward infection in those who are asymptomatic or mildly symptomatic and continue to work. However, vaccination programmes may change the profile of the risks outlined.

HCW infection can create additional problems over and above the infection itself: they can expose hospitalised patients - a more vulnerable population; they may impact the workforce capacity of the NHS at a time of peak demand, particularly with the possiblity of long COVID having a longer-term impact on ability to work; and they provide a route to amplify transmission by seeding infection in the community which can occur via discharging infectious patients and via transmission to contacts of HCWs and patients.

\section{Lessons learnt}

Understanding the dynamics driving the COVID-19 pandemic is essential to improve NHS planning, especially during the winter period, which brought the second wave of COVID-19, and with the potential for additional waves in the future. This study is based on data from the first wave of infection in England and, therefore, may not be representative of the current situation. However, we can learn important lessons from the first wave and there needs to be an intensified focus on strategies to prevent infection and protect both HCWs and patients. Potential strategies include robust, appropriate and ample PPE; education in infection, prevention and control, including hand washing, respiratory hygiene, cleaning of hospital environments and equipment, and donning and doffing (putting on and taking off) PPE; enhanced physical distancing within the hospital environment (especially in staff rest areas, waiting rooms); improving ventilation where possible (eg, increase airflow); cohorting of infected and non-infected patients in different areas; minimising staff movement and circulation between infected and uninfected areas; maximising remote working and consultation where possible; and enhanced testing of staff and patients.

\section{Strengths and limitations}

The key strength of this report is the sample size and the national coverage which gives a thorough and unique picture of seroprevalence in HCWs. The study population is substantially larger than similar reports and while it provides useful insights it also highlights the need for a better surveillance system linked to granular data regarding risk factors (including staff role, clinical location, age, sex, ethnicity) and further prospective studies to be undertaken to allow critical questions to be answered.

This is a technical report and not a research project, meaning there are a number of limitations. Firstly, it is a cross-sectional snapshot of one data time point and therefore, it is unable to address many key questions about the source of infection in NHS staff. Furthermore, the data were not collected for research analysis and this introduces many limitations related to the data and limits the conclusions which can be drawn. For example, there was no specific sampling frame, but given the high coverage rate, these data provide insight on a high proportion of the NHS staff population (although it is unlikely to be fully random or representative); the data are incomplete and there is a lack of associated metadata given the anonymisation; we are unable to confirm de-duplication of the data set, although the study team thought that the number of duplicate results was likely to be so small that it would have minimal effect on the conclusions drawn in a data set this large. These data include all NHS Trust staff, including office workers. Therefore, the exposure risks vary considerably and may skew the results, but we are unable to differentiate between frontline workers or role within the NHS. This data set does not capture the whole health and social care system, such as care homes and primary care. We are also only able to determine tests at a regional level and are unable to investigate trust level variability.

Another important consideration is that this testing programme only used antibody production as a marker for infection. There are many critical immunological questions, and this is a very active area of research, including the role of T-cell immunity in COVID-19 infections. This study, therefore, may underestimate infection as it only uses antibody seroconversion, which may wane over time, and it does not capture any element of T-cell immunity or antibody waning or changes in seroprevalence. Serological testing may also have a lower sensitivity than RT-PCR testing and it would have been useful to 
incorporate contemporaneous RT-PCR data to enable more thorough exploration of NHS staff infections.

We were only able to use a simplistic comparison of seroprevalence between NHS staff and the general population given the data available. However, both data sets use national cohorts, although there are many differences between these populations that may generate bias, including: the sampling frames between the two groups are different; the slightly different sampling timelines; variations in the availability of testing; and the serological assays used may be different (both between the groups and across sites within the NHS cohort) with different test performance characteristics making direct comparisons less robust. ${ }^{33}$

\section{Future research}

Finally, given the data constraints, particularly the lack of metadata, we were unable to undertake any granular analysis or weight the initial analysis presented here. To be able to fully understand the critical issue of infection risk in NHS staff, better surveillance systems and data are urgently required, including linked epidemiological and genomic data which may allow many questions to be answered which would not be addressed by traditional epidemiological data alone, for example, direction of transmission and drivers/risk factors associated with transmission. Furthermore, given the association between infection rate and ethnicity, a study including breakdown of ethnicity is needed to add to this important field of research. The SIREN (SARS-CoV-2 Immunity \& Reinfection Evaluation) Study, a large-scale HCW survey to measure prevalence and incidence, is one such study which will advance our understanding in this field. ${ }^{34}$ This study will collect comprehensive data on HCWs and allow a more detailed analysis to address key concerning questions, including those highlighted by the Royal Society Delve Initiative report on nosocomial infection ${ }^{14}$ : does HCW infection differ by role in hospital, clinical setting, ethnicity, availability of PPE?; what drives infection (eg, staff-to-staff, patient-to-patient, staff-to-patient or patientto-staff transmission)?; where does transmission occur (on wards, theatres, rest areas)?; how does staff movement amplify disease spread?; what fraction of HCW infection can be attributed to occupational infection rather than community acquisition?; and what fraction of community cases are likely seeded from HCW or other hospitalrelated causes? This paper is high level, but the hope is that it stimulates discussion and further work to identify and address the key missing questions.

\section{SUMMARY}

In conclusion, this study provides valuable insights into the dynamics of COVID-19 infection in NHS staff in England at a national level during the first wave of the pandemic. It confirms previous findings (for both COVID-19 and other respiratory infection outbreaks) showing significant increased risk of infection in HCWs and suggests that NHS staff are at higher risk of acquiring infection than the general population. Additional future research is needed to address key questions and should focus on understanding the drivers of transmission, the identification of high-risk subgroups among HCWs and how infection is spread (eg, mechanism and direction of transmission) within hospital settings. Genomic studies and adequately powered, prospective cohort studies will play a key role. Prevention of infection in both HCWs and patients is possible with optimal infection prevention and control practices and could have a substantial impact on the scale and resurgence of future waves of COVID19. NHS planning for subsequent waves of COVID-19 in England must include a focus on the development of targeted strategies to minimise nosocomial infection and protect both NHS staff and patients. This is especially important given the extra pressures on the NHS during winter.

Acknowledgements We would like to thank and pay tribute to the many NHS staff who have taken part in the testing programme and have worked so tirelessly, at considerable personal risk, during the current COVID-19 pandemic. We also thank NHS England and Improvement (NHS E\&l) and ONS for their hard work, particularly collecting, processing and sharing the data used in this report, notably Melanie Matthews, Jon Couchman and Nicola Hunt (from NHS E\&l); and Ruth Studley, Dominic Brown, Heledd Thomas, Tristan Pett, and Joe Jenkins (from ONS). Finally, we would like to thank Paul Moore, Department of Health and Social Care statistician, from the Coronavirus Analytical Coordination Hub for his advice and input on the analysis; Prof Dame Anne Johnson, Institute for Global Health (UCL), for her advice and comments on the article; and the Office of the Chief Medical Officer for England (DHSC) for their support.

Contributors AF conceptualised the idea, together with NHS E\&I colleagues. The data acquisition and cleaning from NHS E\&I was led by DW, with support from Melanie Matthews and team; and from ONS was led by ES with support from Ruth Studley. CEMC undertook the analysis and interpretation with support from DHSC statisticians (Paul Moore). CEMC developed the manuscript with input from AF, ES and DW. All authors read and approved the final version of the manuscript, in addition to several other contributors from NHS E\&I and ONS. All authors are accountable for this work and will ensure that any questions related to the accuracy or integrity of this work are appropriately investigated and resolved.

Funding This work was undertaken as part of the routine evaluation of the NHS staff testing programme. CEMC is funded by an NIHR Clinical Lecutreship.

Disclaimer AF, the lead author (and manuscript's guarantor), affirms that the manuscript is an honest, accurate, and transparent account of the study being reported; that no important aspects of the study have been omitted; and that any discrepancies from the study as originally planned have been explained.

Competing interests None declared.

Patient and public involvement Patients and/or the public were not involved in the design, or conduct, or reporting, or dissemination plans of this research.

Patient consent for publication Not required.

Provenance and peer review Not commissioned; externally peer reviewed.

Data availability statement № data are available. No additional data are available.

Open access This is an open access article distributed in accordance with the Creative Commons Attribution Non Commercial (CC BY-NC 4.0) license, which permits others to distribute, remix, adapt, build upon this work noncommercially, and license their derivative works on different terms, provided the original work is properly cited, appropriate credit is given, any changes made indicated, and the use is non-commercial. See: http://creativecommons.org/ licenses/by-nc/4.0/.

ORCID iD

Cordelia E M Coltart http://orcid.org/0000-0003-0176-8831 


\section{REFERENCES}

1 WHO. Who coronavirus disease (COVID-19) Dashboard, 2020. Available: https://covid19.who.int/ [Accessed 9 Nov 2020].

2 UK Government. Coronavirus (COVID-19) in the UK, 2020. Available: https://coronavirus.data.gov.uk/details/cases [Accessed 9 Nov 2020].

3 WHO. Summary of probable SARS cases with onset of illness from 1 November 2002 to 31 July 2003. Geneva, Switzerland: WHO, 2003.

4 Peck AJ, Newbern EC, Feikin DR, et al. Lack of SARS transmission and U.S. SARS case-patient. Emerg Infect Dis 2004;10:217-24.

5 Ruan YJ, Wei CL, Ee AL, et al. Comparative full-length genome sequence analysis of 14 SARS coronavirus isolates and common mutations associated with putative origins of infection. Lancet 2003;361:1779-85.

6 Lee N, Hui D, Wu A, et al. A major outbreak of severe acute respiratory syndrome in Hong Kong. N Engl J Med 2003;348:1986-94.

7 Lietz J, Westermann C, Nienhaus A, et al. The occupational risk of influenza $\mathrm{A}(\mathrm{H} 1 \mathrm{~N} 1)$ infection among healthcare personnel during the 2009 pandemic: a systematic review and meta-analysis of observational studies. PLoS One 2016;11:e0162061-e.

8 Kuster SP, Shah PS, Coleman BL, et al. Incidence of influenza in healthy adults and healthcare workers: a systematic review and meta-analysis. PLoS One 2011;6:e26239-e.

9 Kilmarx PH, Clarke KR, Dietz PM, et al. Ebola virus disease in health care workers--Sierra Leone, 2014. MMWR Morb Mortal Wkly Rep 2014;63:1168-71.

10 Coltart CEM, Johnson AM, Whitty CJM. Role of healthcare workers in early epidemic spread of Ebola: policy implications of prophylactic compared to reactive vaccination policy in outbreak prevention and control. BMC Med 2015;13:271.

11 Zheng L, Wang X, Zhou C, et al. Analysis of the infection status of healthcare workers in Wuhan during the COVID-19 outbreak: a cross-sectional study. Clin Infect Dis 2020;71:2109-13.

12 Nguyen LH, Drew DA, Graham MS, et al. Risk of COVID-19 among front-line health-care workers and the general community: a prospective cohort study. Lancet Public Health 2020;5:e475-83.

13 Rudberg A-S, Havervall S, Månberg A, et al. SARS-CoV-2 exposure, symptoms and seroprevalence in healthcare workers in Sweden. Nat Commun 2020;11:5064.

14 Royal Society: The Delve Initiative. Scoping report on hospital and health care acquisition of COVID-19 and its control. London, UK: Royal Society, 2020.

15 Rickman HM, Rampling T, Shaw K. Nosocomial transmission of COVID-19: a retrospective study of 66 hospital-acquired cases in a London teaching hospital. Clinical infectious diseases : an official publication of the Infectious Diseases Society of America 2020:ciaa816.

16 Taylor J, Rangaiah J, Narasimhan S, et al. Nosocomial COVID-19: experience from a large acute NHS trust in south-west London. $J$ Hosp Infect 2020;106:621-5.
17 Carter B, Collins JT, Barlow-Pay F, et al. Nosocomial COVID-19 infection: examining the risk of mortality. The COPE-Nosocomial study (COVID in older people). J Hosp Infect 2020;106:376-84.

18 Peeling RW, Wedderburn CJ, Garcia PJ, et al. Serology testing in the COVID-19 pandemic response. Lancet Infect Dis 2020;20:e245-9.

19 NHS England. Personal communication 2020.

20 Office for National Statistics. COVID-19 infection survey, 2020. Available: https://www.ons.gov.uk/peoplepopulationandcommunity/ healthandsocialcare/conditionsanddiseases/bulletins/coronavirusc ovid19infectionsurvey/england10may2020\#covid-19-infection-survey [Accessed 9th Sep 2020].

21 Houlihan CF, Vora N, Byrne T, et al. Pandemic peak SARS-CoV-2 infection and seroconversion rates in London frontline health-care workers. Lancet 2020;396:e6-7.

22 Hunter E, Price DA, Murphy E, et al. First experience of COVID-19 screening of health-care workers in England. Lancet 2020;395:e77-8.

23 Hunt J. Twitter 2020.

24 Jones NK, Rivett L, Sparkes D, et al. Effective control of SARSCoV-2 transmission between healthcare workers during a period of diminished community prevalence of COVID-19. Elife 2020;9. doi:10.7554/eLife.59391. [Epub ahead of print: 19 Jun 2020].

25 Pallett SJC, Rayment M, Patel A, et al. Point-Of-Care serological assays for delayed SARS-CoV-2 case identification among healthcare workers in the UK: a prospective multicentre cohort study. Lancet Respir Med 2020;8:885-94.

26 Shields A, Faustini SE, Perez-Toledo M, et al. SARS-CoV-2 seroprevalence and asymptomatic viral carriage in healthcare workers: a cross-sectional study. Thorax 2020;75:1089-94.

27 Eyre DW, Lumley SF, O'Donnell D, et al. Differential occupational risks to healthcare workers from SARS-CoV-2 observed during a prospective observational study. Elife 2020;9. doi:10.7554/ eLife.60675. [Epub ahead of print: 21 Aug 2020].

28 Iversen K, Bundgaard H, Hasselbalch RB, et al. Risk of COVID-19 in health-care workers in Denmark: an observational cohort study. Lancet Infect Dis 2020;20:1401-8.

29 Sandri MT, Azzolini E, Torri V. Igg serology in health care and administrative staff populations from 7 Hospital representative of different exposures to SARS-CoV-2 in Lombardy, Italy. medRxiv 2020.

30 Rivett L, Sridhar S, Sparkes D, et al. Screening of healthcare workers for SARS-CoV-2 highlights the role of asymptomatic carriage in COVID-19 transmission. Elife 2020;9. doi:10.7554/eLife.58728. [Epub ahead of print: 1105 2020].

31 Evans S, Agnew E, Vynnycky E. The impact of testing and infection prevention and control strategies on within-hospital transmission dynamics of COVID-19 in English hospitals 2020.

32 Public Health England. Personal communication.2020.

33 Whitman JD, Hiatt J, Mowery CT, et al. Evaluation of SARS-CoV-2 serology assays reveals a range of test performance. Nat Biotechnol 2020;38:1174-83.

34 Public Health England. Siren study, 2020. Available: https:// snapsurvey.phe.org.uk/siren/ [Accessed 31 Dec 2020]. 